\title{
EUV Spectra of the Full Solar Disk: Analysis and Results of the Cosmic Hot Interstellar Plasma Spectrometer (CHIPS)
}

\author{
M.M. Sirk • M. Hurwitz • W. Marchant
}

Received: 12 January 2010 / Accepted: 14 June 2010 / Published online: 14 July 2010

(C) The Author(s) 2010. This article is published with open access at Springerlink.com

\begin{abstract}
We analyze EUV spectra of the full solar disk from the Cosmic Hot Interstellar Plasma Spectrometer (CHIPS) spanning a period of two years. The observations were obtained via a fortuitous off-axis light path in the 140-275 A passband. The general appearance of the spectra remained relatively stable over the two-year time period, but did show significant variations of up to $25 \%$ between two sets of Fe lines that show peak emission at $1 \mathrm{MK}$ and $2 \mathrm{MK}$. The variations occur at a measured period of 27.2 days and are caused by regions of hotter and cooler plasma rotating into, and out of, the field of view. The CHIANTI spectral code is employed to determine plasma temperatures, densities, and emission measures. A set of five isothermal plasmas fit the full-disk spectra well. A 1-2 MK plasma of Fe contributes $85 \%$ of the total emission in the CHIPS passband. The standard Differential Emission Measures (DEMs) supplied with the CHIANTI package do not fit the CHIPS spectra well as they over-predict emission at temperatures below $\log _{10} T=6.0$ and above $\log _{10} T=6.3$. The results are important for cross-calibrating TIMED, SORCE, SOHO/EIT, and CDS/GIS, as well as the recently launched Solar Dynamics Observatory.
\end{abstract}

Keywords Corona $\cdot$ Solar EUV irradiance $\cdot$ Spectral line, intensity and diagnostics

\section{Introduction}

The solar EUV irradiance is the primary source of energy and ionization of the Earth's upper atmosphere. Variations in the EUV flux are responsible for dramatic changes in the density of neutrals and ions, affecting satellite drag forces in near-Earth orbit by up to a factor of ten and modifying the propagation of radio frequency signals (Fuller-Rowell et al., 2004).

M.M. Sirk $(\bowtie) \cdot$ M. Hurwitz · W. Marchant

Space Sciences Laboratory, University of California, Berkeley, CA 94720, USA

e-mail: sirk@ssl.berkeley.edu

M. Hurwitz

e-mail: markh@ssl.berkeley.edu

W. Marchant

e-mail: marchant@ssl.berkeley.edu 
Solar high spectral-resolution EUV spectra were first obtained via sub-orbital rocket flights (Behring, Cohen, and Feldman, 1972; Malinovsky and Heroux, 1973; Thomas and Neupert, 1994), and SkyLab (Dere, 1978). Collectively, these early missions discovered over 400 emission lines from 17 elements between $50 \AA$ and $600 \AA$, indicating the presence of a 1 to $2 \mathrm{MK}$ plasma dominated by emission from Fe.

The Solar and Heliospheric Observatory/EUV Imaging Telescope (SOHO/EIT) obtains full-disk images in four $\approx 10 \AA$ (FWHM) passbands centered at 171, 195, 285, and $304 \AA$. These pictures show where EUV emission originates; however, as concluded by Hock and Eparvier (2008), it is essentially impossible to accurately convert the broad-band photometry to physical units without simultaneous knowledge of the solar spectrum.

SOHO/CDS/GIS has one channel (150 to $220 \AA$ ) that overlaps CHIPS, but shows spectral "ghosts" and temporal variations in throughput that require careful calibration (Kuin and Del Zanna, 2007) to provide accurate line intensities and ratios.

The Hinode/Extreme Ultraviolet Spectrograph (EIS) is a high spectral- and spatialresolution instrument that provides very detailed temperature and density maps, but can only see a small strip ( 1 or $2^{\prime \prime}$ wide by $512^{\prime \prime}$ long) of the Sun (Young et al., 2009).

Each of these instruments has its advantages and limitations. At one extreme are the high spectral- and spatial-resolution spectrographs that are not well suited for total solar irradiance; at the other are the broad-band photometers which, even if accurately calibrated, cannot provide details of the solar spectrum. Rocket flights typically provide only a fourminute snapshot, and thus cannot address temporal variations.

Great progress in determining the EUV contribution to total solar irradiance has been made by the Thermosphere, Ionosphere, Mesosphere, Energetics, and Dynamics (TIMED), and Solar Radiation and Climate Experiment (SORCE) missions (Woods, 2008). The daily data provided by these instruments are well calibrated, broad-band (width 70 to $100 \AA$ ) photodiode measurements, which are used to scale reference CHIANTI (Landi et al., 2006; Dere et al., 1997) model spectra (quiet Sun, active region, coronal hole, and flare) that depend upon assumed Differential Emission Measures (DEMs) and out-of-band proxies. We refer to the SORCE XPS Level 4 Version 10 model (outlined in detail in Woods et al., 2008) as XPSL4 hereafter.

Here we present full-disk solar spectra from the Cosmic Hot Interstellar Plasma Spectrometer (CHIPS) covering a period of two years. The data from the SORCE, TIMED/SEE, and SOHO/EIT missions complement those of CHIPS. The first two provide the total irradiance, SOHO/EIT shows where the EUV emission originates, and CHIPS provides spectra from which temperatures and densities may be determined as well as provide the spectral shape between 140 and $270 \AA$ required to improve the calibration of the contemporaneous SORCE/XPS and TIMED/SEE photodiode measurements, and the SOHO/EIT broadband full-disk images. A recent flight of the rocket prototype Solar Dynamics Observatory (SDO)/Extreme Ultraviolet Variability Experiment (EVE) (Woods et al., 2009) provided an EUV spectrum with similar passband and resolution as CHIPS. This spectrum is utilized to cross-check the quality of the solar observations and the calibration of the CHIPS throughput. An uncalibrated, mean CHIPS spectrum was first presented by Hurwitz et al. (2006), and compared to laboratory plasmas by Lepson et al. (2008).

In this work we explore to what extent the solar corona can be described by a set of simple plasmas, and how these may be used in modeling the spectral shape of the solar irradiance. We present both raw and flux calibrated spectra, quantify the spectrometer's response to offaxis solar illumination, perform CHIANTI plasma modeling, and outline the contents of a publicly available archive. 
Figure 1 Schematic showing relative locations of the six slit plates and diffraction gratings employed by CHIPS. Left: central channel. Middle: non-mirrored channels. Right: non-mirrored and mirrored channels (rear row).
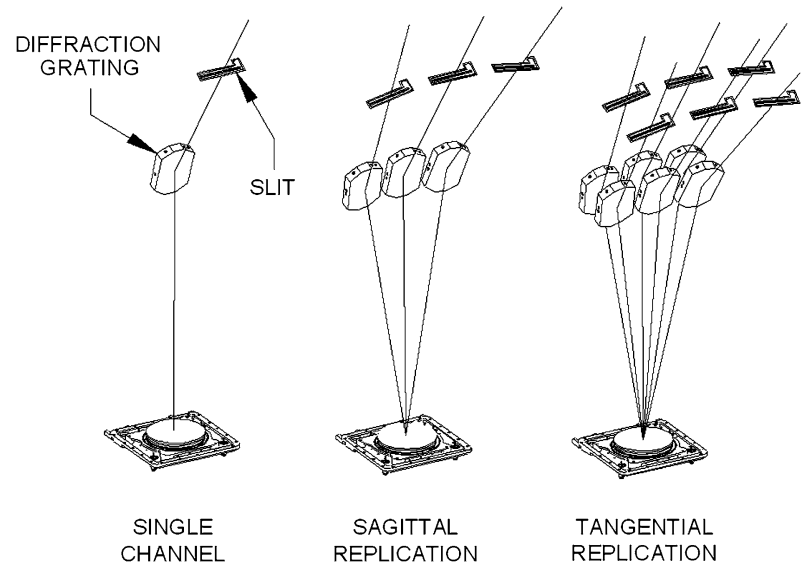

TANGENTIAL REPLICATION

\section{CHIPS Instrument}

The CHIPS satellite is a NASA University Explorer mission devoted to diffuse background spectroscopy of the interstellar medium at moderate resolution $(\lambda /(\Delta \lambda \mathrm{FWHM}) \approx 120)$ in the EUV passband 90-260 A. NASA launched CHIPS from Vandenberg AFB on 12 January 2003 as a secondary payload on a Delta II rocket into a circular polar orbit with an inclination of $94^{\circ}$ and an altitude of $590 \mathrm{~km}$.

The three-axis stabilized satellite consists of a 35-kg spacecraft built by SpaceDev, Inc., and a $25-\mathrm{kg}$ science instrument provided by the University of California, Berkeley. The spectrograph consists of a micro-channel plate detector placed at the focus of six varied-linespacing cylindrically curved diffraction gratings. Mounted $8.8 \mathrm{~mm}$ in front of the detector face is a filter frame which holds the vacuum deposited $\mathrm{Al}, \mathrm{Zr}$, and polyamide - boron thin film $(\approx 1000 \AA$ thick) filters, which suppress higher diffracted orders as well as longerwavelength scattered and stray light. Unlike traditional imaging spectrographs, the CHIPS design does not employ a telescope to gather light. Regardless of a source's position within the field of view, light of a particular wavelength is directed at a particular dispersion angle to the micro-channel plate detector. A single spectrum of the entire $4^{\circ} \times 26^{\circ}$ field of view may thus be obtained simultaneously. The opto-mechanical design of the instrument is outlined in Sholl et al. (2003), the detector characteristics in Marckwordt et al. (2003), the calibration and in-flight performance in Sirk et al. (2003), and the primary mission science results in Hurwitz, Sasseen, and Sirk (2005).

Each diffraction grating is equipped with a pair of adjacent, fixed, entrance slits, 0.25 and $1.0 \mathrm{~mm}$ wide (Figure 1). In front of each slit pair is a rotary mechanism that allows diffuse light to illuminate the grating through one slit or the other (or can be set to block both). Between the slits and three of the gratings are flat grazing-incidence mirrors that serve to coalign the field of view of those spectral channels with their non-mirrored counterparts (see Figure 2, right). During normal science observations, the instrument boresight is constrained to be far from the Sun, so that the rotary mechanism and entrance slits are shaded from direct sunlight. When several comets presented themselves as targets of opportunity (Sasseen et al., 2006), we relaxed this constraint and allowed the instrument boresight to encroach within tens of degrees of the Sun. The resulting spectra showed features that seemed not to originate from the comets, were far too bright to arise in the interstellar medium, and did not (without shifting) match the reflected solar spectrum that had been recorded during observations of the full Moon. 

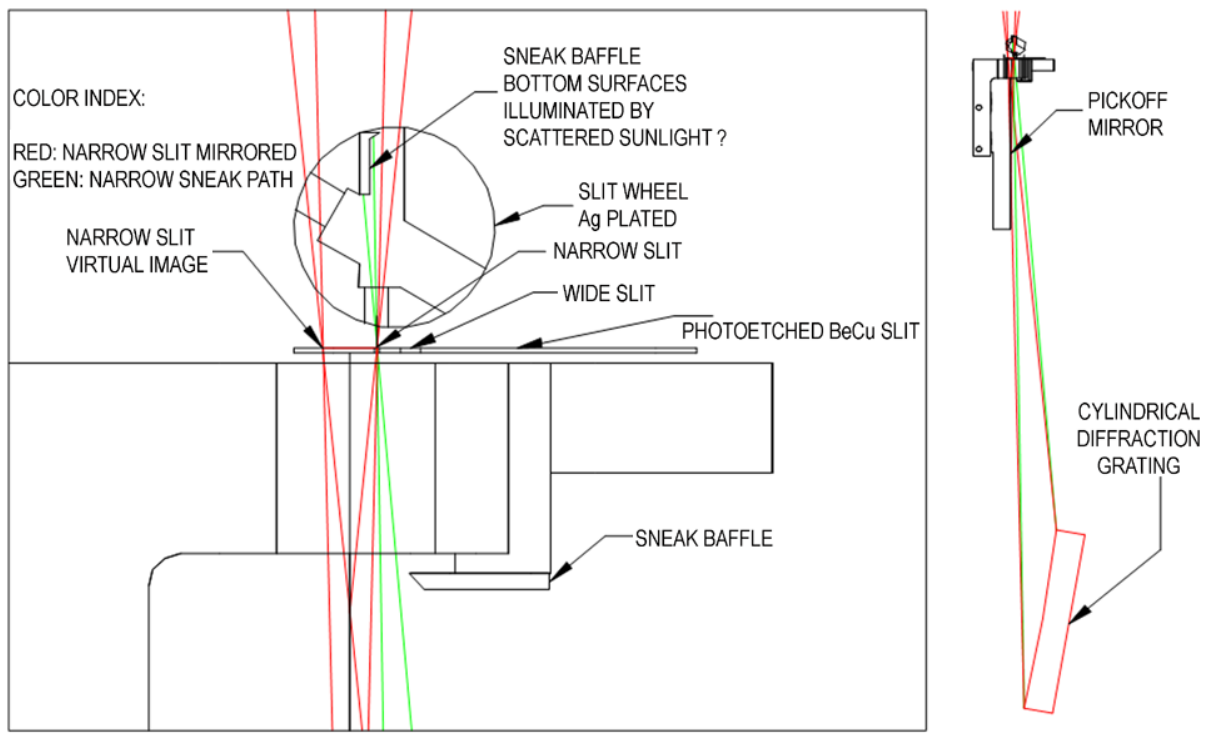

Figure 2 Edge-on views of slit and slit-wheel shutter mechanism showing direct and scattered light paths. Left: field of view of direct path and virtual image of direct path (red), and field of view of scattered path off baffle (green). Right: general view showing location of slit wheel, pickoff mirror, and diffraction grating. Notice that the scattered path does not intersect the pickoff mirror.

We ultimately discovered that in certain geometries, scattered sunlight could pass through an entrance slit, avoid the flat mirror, and strike a diffraction grating at an angle different from the nominal $14^{\circ}$ incidence. Ray-trace analysis by us and Sholl (2005) confirmed that light scattered from the back side of a baffle could follow this path to the detector, producing a spectrum shifted by $\approx 11 \AA$ from the nominal wavelength solution (see Figure 2, left). After much experimentation with the Sun angle and slit orientation (angle of slit axis with respect to the ecliptic plane), a configuration was found that nominally showed a single-channel solar spectrum. We refer to this path as the "light leak." The light leak has no adverse effect on ordinary science observations. Its throughput is six orders of magnitude lower than for emission within the direct field of view, and diffuse emission comprising the main science target is so faint as to be barely detectable. The light leak in this case provides a serendipitous way of measuring the EUV spectrum of light integrated from the solar disk.

\section{Observations}

The CHIPS satellite performed 1450 observations of the Sun from 03 April 2006 to 05 April 2008, a period of generally declining activity following the solar maximum of 2001. The satellite was decommissioned on 14 April 2008. Typical observations ranged from 1 to 15 minutes which yielded spectra of roughly $10^{5}$ total counts each. Occasionally, up to 15 such observations per day were performed. However for a variety of causes, including passages through the South Atlantic Magnetic Anomaly, passages through the electron belts at high magnetic latitudes, targets of opportunity, precession of the orbit into full sunlight, and satellite shutdowns, the cadence of observations is far from uniform.

We present raw spectra integrated over the two-year period for the upper $\mathrm{Zr} / \mathrm{Al}$ filters, and the lower large Al filter in Figure 3. The brightest features are from Fe IX through 
Figure 3 Raw CHIPS spectra binned at $0.11 \AA$ for the $\mathrm{Zr} / \mathrm{small}$ Al filter (top) and the large $\mathrm{Al}$ filter (bottom). Wavelength regions of filter-frame occlusion and second-channel contamination (described in Section 4.3) features are indicated.
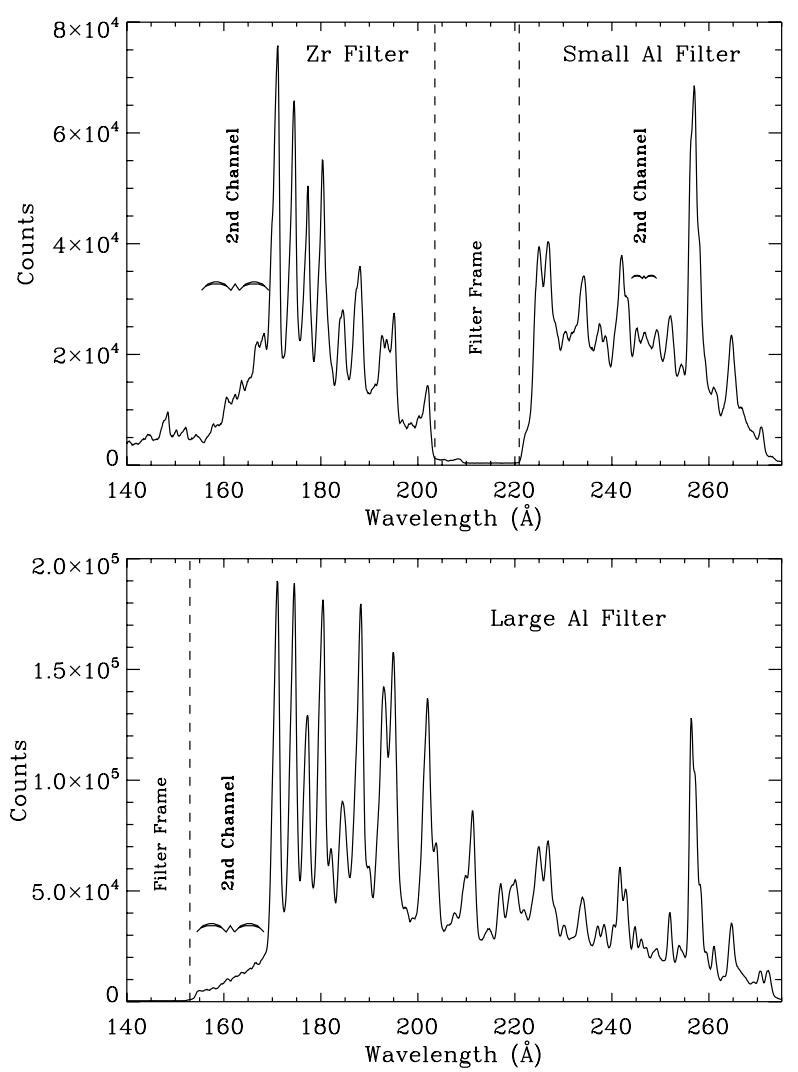

Fe XV. Also evident is a continuum of in-band scattered light from the diffraction gratings superimposed with second- (and possibly third-) channel features (discussed in Section 4.3), which occur more frequently, and to a greater degree, in the upper $\mathrm{Zr} / \mathrm{Al}$ filter. Particle background (primarily high-energy electrons) is negligible as evinced by the near zero count rates in the shadows of the filter frames.

The general appearance of the daily spectra remained relatively stable. The emission lines labeled in Figures 4 and 5 did not appear and disappear, but were visible at all times, even during the quiet-Sun period of April 2008. During 2006, CHIPS observed an M-class flare (27 April 2006 15:22 UT M7.9), and just missed a second one by 24 hours (06 July 2006 08:13 UT M2.5). Spectra extracted in the hours just prior to and during the flares showed no discernible differences in line ratios, indicating that moderate intensity flares do not necessarily produce large changes in the shape of the EUV spectrum. We present a more detailed analysis in Section 5.6.

\section{Calibrations}

\subsection{Calibration Strategy}

Each observation is processed by our standard science pipeline and spectra extracted as described by Hurwitz, Sasseen, and Sirk (2005). Because the solar data are obtained via 


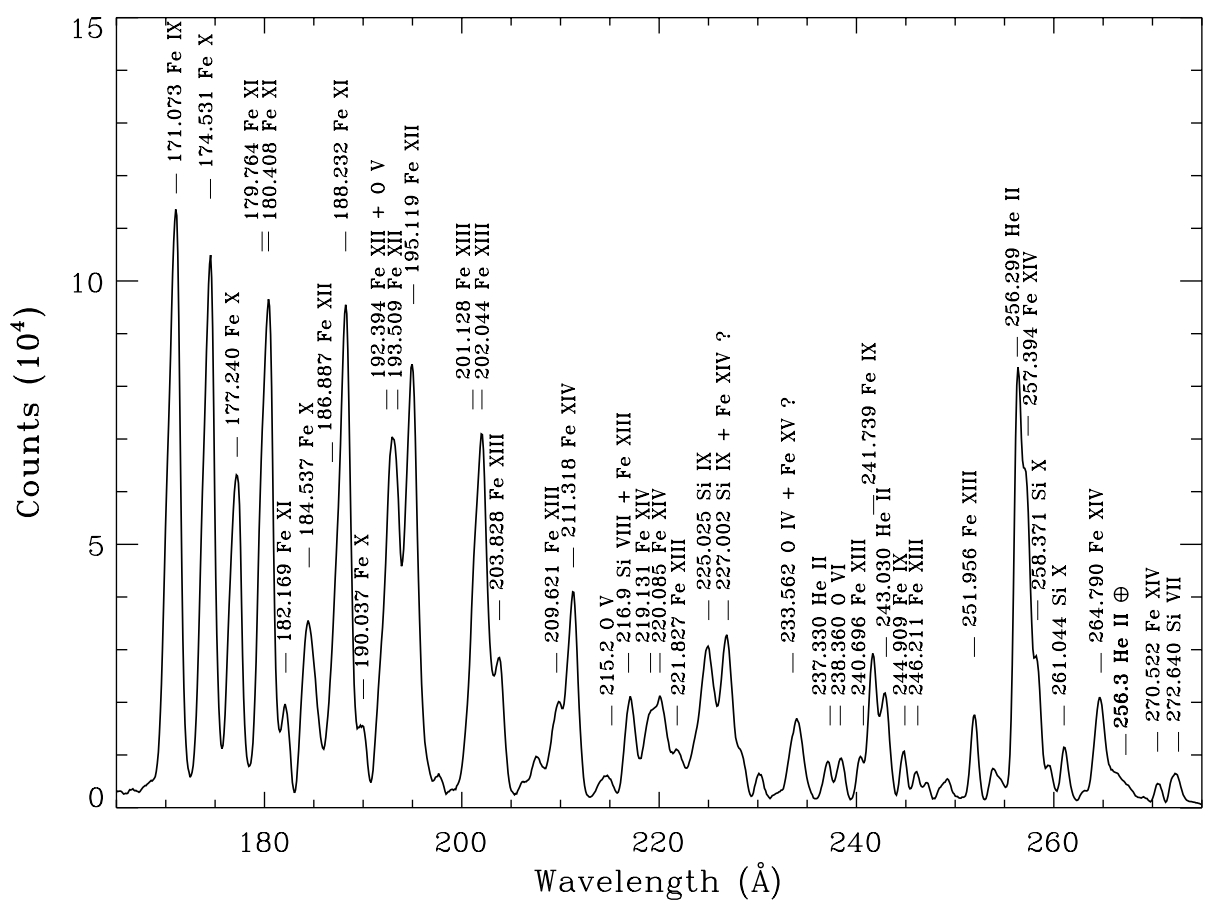

Figure 4 Cleaned, time-averaged, background-subtracted Al-filter spectrum binned at 0.11 Å. Line identifications and wavelengths are from the CHIANTI database (Landi et al., 2006; Dere et al., 1997).

the fortuitous light leak, the ground-based calibrations for wavelength scale and throughput are not directly applicable. To analyze the spectra quantitatively, the instrument required an ad hoc recalibration using the solar data itself. The strategy is one of iteration: as various instrumental effects become known, the entire data set is reprocessed to produce the highestquality spectra.

\subsection{Reference Spectra}

The first step is to create a provisional reference spectrum for each filter set by simply adding up all the observations. Next, each individual spectrum is shifted in the dispersion direction until it best matches (by cross correlation) the provisional reference spectrum, thus placing all data onto a common dispersion grid. The shifts proved to be minor, typically 0 to $0.2 \AA$, demonstrating that the spectrograph is essentially free from flexure problems. Finally, all of the shifted spectra are co-added a second time to create improved reference spectra for both the Al-filter and the $\mathrm{Zr} / \mathrm{Al}$-filter panels.

\subsection{Multiple-Channel Contamination}

In spite of our efforts to best configure the satellite orientation to produce a single-channel spectrum, multiple-channel contamination is still evident in the majority of pointings and is quite variable in intensity from one observation to the next. The contamination is not the result of second-order grating features, but rather, is caused by scattered light from a second (or third) slit and grating pair striking the detector (see Figure 1). The contamination 


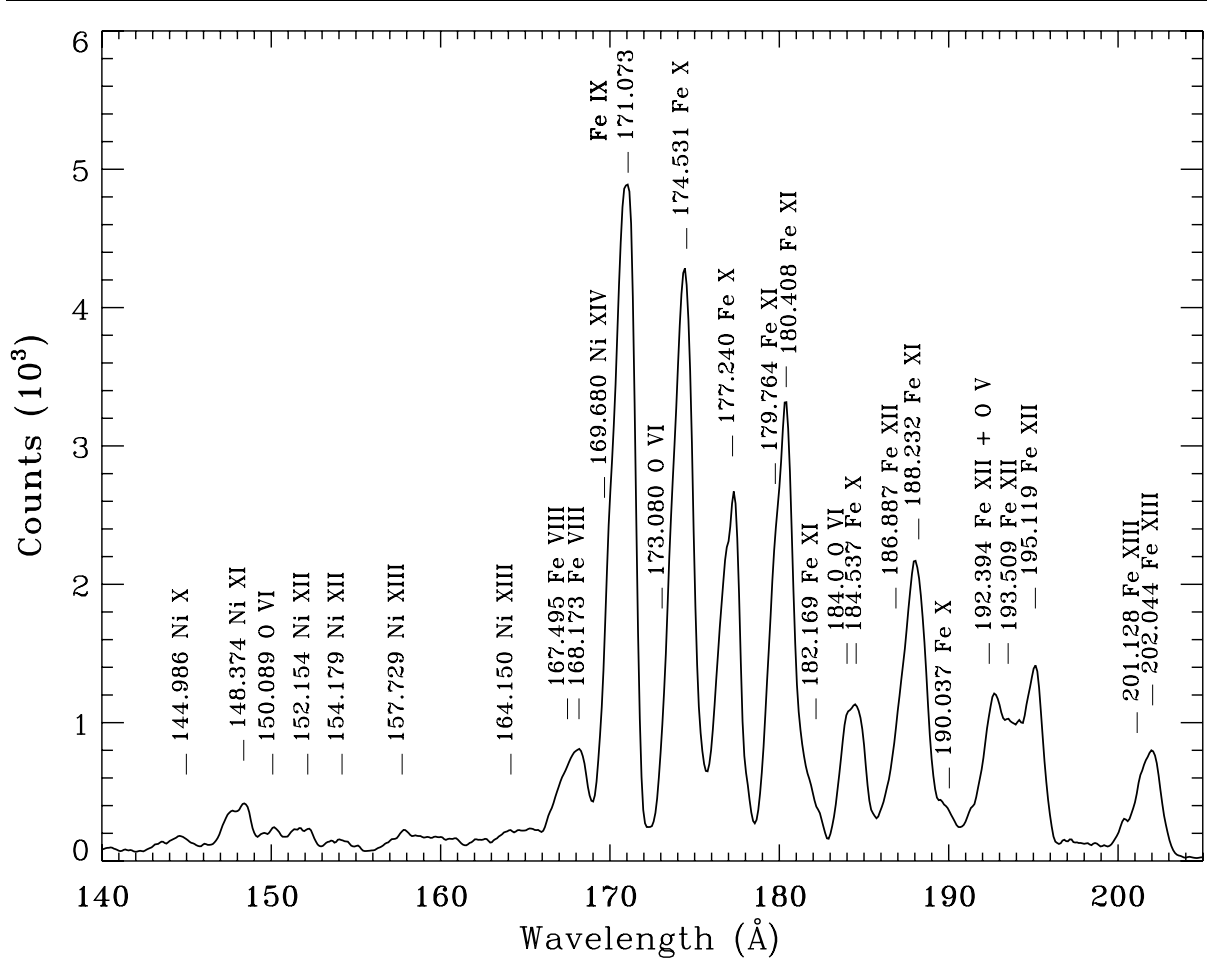

Figure 5 Cleaned time-averaged, background-subtracted Zr-filter spectrum binned at $0.11 \AA$ A. Line identifications for Ni X and Ni XI are from Mewe, Gronenschild, and van den Oord (1985).

is manifest as additional features shortward of Fe IX $171.073 \AA$ in Figure 3 in both the $\mathrm{Zr}$ and $\mathrm{Al}$ filters. The likely cause for the observed variations is that small errors in the pointing knowledge of the satellite of $\approx 1.0^{\circ}$ lead to large changes in the illumination of the slits, with each channel responding differently with regard to wavelength scale and throughput. Efforts to decouple the channels proved futile. We found, for example, that a multi-channel spectrum is not simply the linear combination of scaled and shifted reference spectra, even if the reference spectrum is constructed from contamination-free spectra.

Fortunately, the degree of second-channel contamination is in general small and is easily quantified by comparing count rates on either side of the absorption edge of the Al filter at $170 \AA$. The effective area of the $\mathrm{Al}$ filter determined by ground calibrations (Sirk et al., 2003) show that the sensitivity just shortward of $170 \AA$ is about $13 \%$ of the $170-180 \AA$ region. Since second-channel contamination is shifted bluewards relative to the "good" channel, any measured flux shortward of $170 \AA$ that is greater than expected is a direct indication of contamination. We calculate a contamination fraction for each observation as the ratio of the average counts per $\AA$ between $155 \AA$ and $167 \AA$ divided by the average counts per $\AA$ of the four brightest iron lines Fe IX 171.073 $\AA$, Fe X $174.531 \AA$, Fe XI $180.408 \AA$, and Fe XI $188.232 \AA$ for the $\mathrm{Al}$ filter. The contamination ranges from 0 to $41 \%$ with a median value of $6 \%$. By selecting appropriate contamination thresholds, the unwanted light may be greatly reduced, or even eliminated, albeit at the expense of rejecting a portion of the observations. For the lightcurve, line ratio, and line-intensity correlation analysis described in Section 5.1, we choose a threshold of $12 \%$, which results in a rejection of about $40 \%$ of the observations. This is a compromise between accepting a small amount of unwanted light 
and rejecting large quantities of the data. For the CHIANTI plasma modeling (Section 5.3), we apply a more stringent threshold of $8 \%$ when fitting the $\mathrm{Zr}$ filter data, since they show a greater degree of contamination, and because there are $\mathrm{Ni}$ emission features of critical interest shortward of $170 \AA$.

\subsection{Background Subtraction}

The CHIPS solar-spectra background is dominated by in-band scattering from the diffraction gratings. High-energy particles, second-order features, and out-of-band scattering from geocoronal emission lines are all negligible. Initial mean backgrounds are determined from the reference spectra by simply drawing by hand. Provisional CHIANTI model fits (specifically outlined in Section 5.3) are performed and the backgrounds adjusted until reasonable fits are found. In Figures 4 and 5 we present the time-averaged background-subtracted spectra with negligible second-channel contamination for the $\mathrm{Al}$ and $\mathrm{Zr}$ filters, respectively. These filtered spectra along with their respective backgrounds are saved as new reference spectra, and the steps outlined in Sections 4.2 and 4.3 repeated once more to create a database with all spectra on a common grid. For analysis, spectral subsets may be easily created by selections based on date, count rate, contamination fraction, line ratios, exposure time, etc.

\subsection{Wavelength Scale}

The dispersion of the CHIPS spectrograph is known to be nearly linear (Sirk et al., 2003) with an average value of $0.11 \AA$ per pixel. Ray-trace analysis also shows that small translations of the slit position cause nearly constant shifts in wavelength of spectral features. Because the solar-spectra light leak is along a unforeseen path, the observed spectral shifts are effectively caused by a slit translation. Thus, the solar wavelength dispersion is also nearly linear, and is shifted about $11 \AA$ bluewards relative to the ground-based wavelength solution. Twenty lines are identified in the $\mathrm{Al}$ and $\mathrm{Zr} / \mathrm{Al}$ cleaned reference spectra using the CHIANTI V5.2.1 database (Landi et al., 2006; Dere et al., 1997) and a quadratic fit performed to determine wavelength as a function of detector $x$ position. Each fit shows an RMS residual of $0.08 \AA$. The resolution of the reference-spectral features is about $1.2 \AA$ FWHM, or $R=140$ at $170 \AA$.

\section{Analysis}

The CHIPS EUV spectra are for the full solar disk, and thus a melange of emission from active regions, quiet Sun, small flares, and coronal holes. Both temperature and density in these plasmas range over two orders of magnitude. In Section 5.1, we investigate the observed temporal variations from one observation to the next and compare the CHIPS data to the sunspot number, and the SORCE XPS L4 V10 spectral model flux (described in Section 1). In Section 5.3 we determine values for temperature and density that best describe the full-disk for time-averaged spectra.

\subsection{Lightcurves, Line Ratios, and Correlations}

The integrated Al-filter count rate is determined over the passband $170-273 \AA$ and is plotted for the two-year period as Figure 6 (upper panel). The median count rate is $447 \mathrm{ct} \mathrm{s}^{-1}$, $+155,-135$. The large scatter from one observation to the next is greater than the variations 

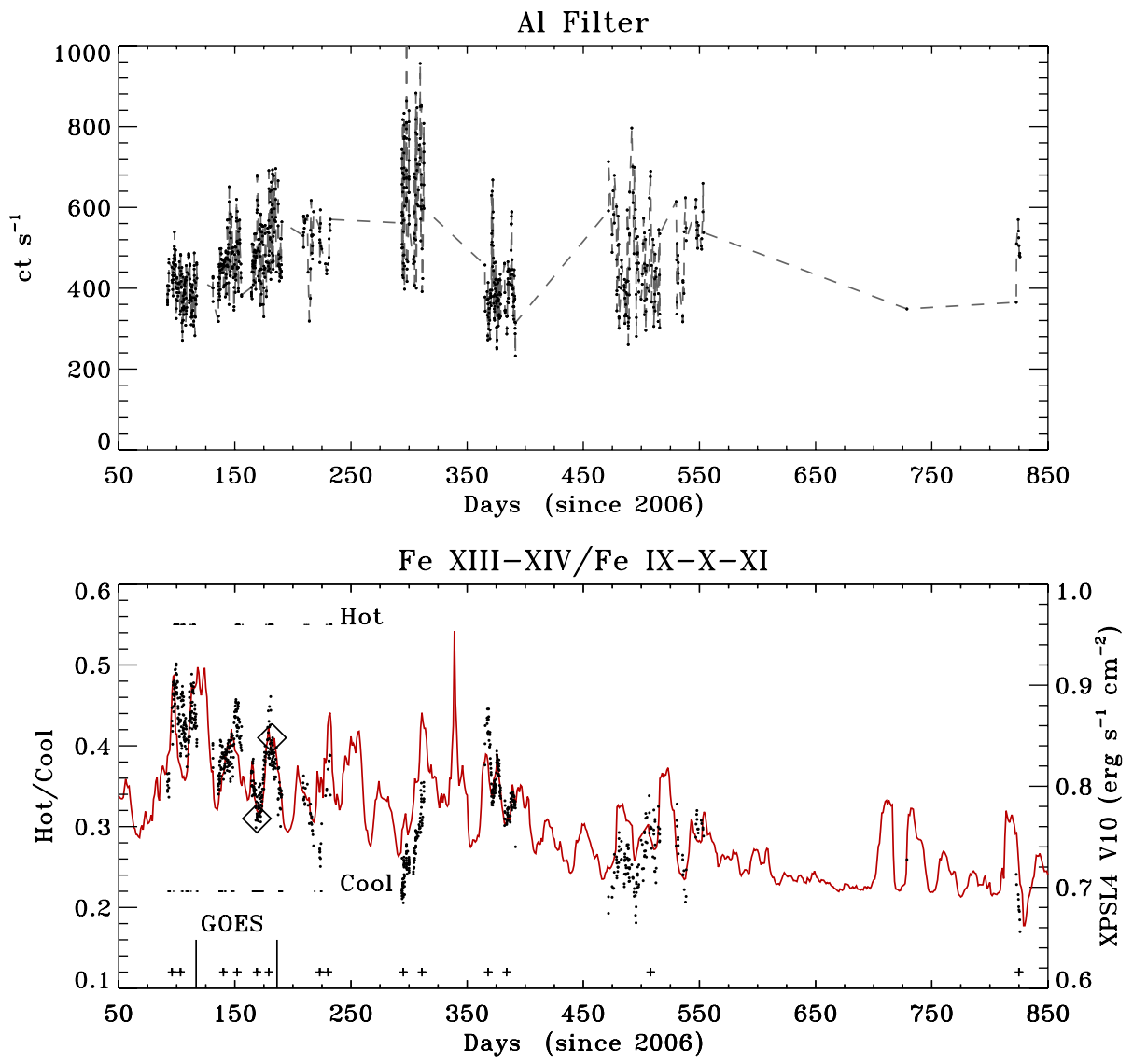

Fe XIII 203/202

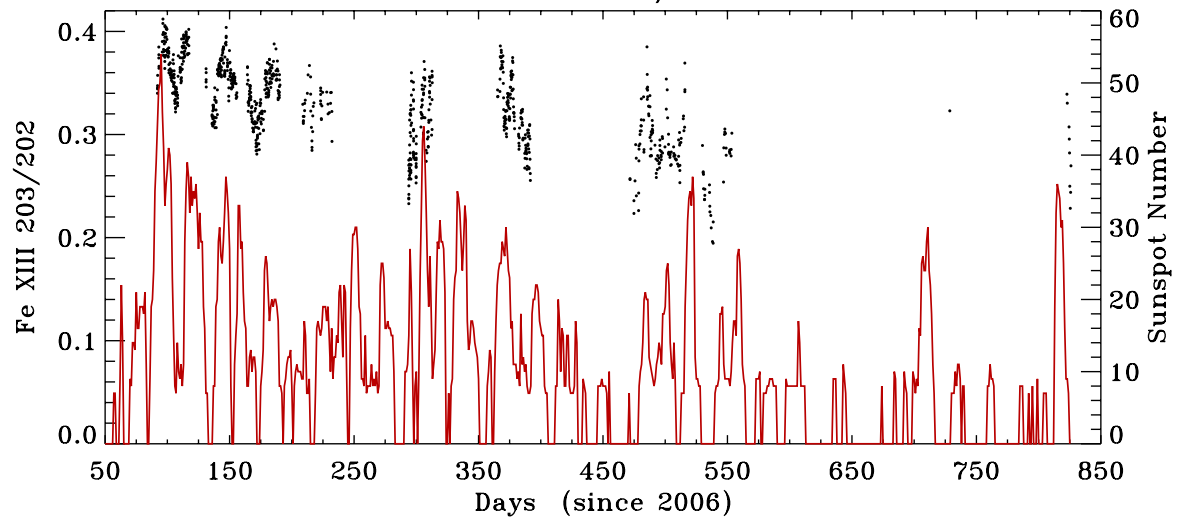

Figure 6 Top: Integrated Al-filter lightcurve (170-273 Å). Middle: Hot-to-cool ratio (dots) compared to SORCE XPSL4 model flux integrated over the CHIPS passband (red line). The diamonds denote the two extreme times chosen for the SOHO/EIT images depicted in Figure 9. Horizontal dashes denote the times from which the hot (upper dashes) and cool (lower dashes) spectra were created. The times of the two GOES flares analyzed in Section 5.6 are marked with vertical dashes. The times corresponding to the extreme-case hot and cool spectra atlas (Figure 10) are demarcated with + symbols. Bottom: CHIPS density-sensitive Fe XIII 203.828 ̊̊/Fe XIII 202.044 ̊̊ line ratio (dots) and the International Sunspot Number (red line). For all three panels, only observations showing less than $12 \%$ multi-channel contamination are shown. 
seen in the integrated XPSL4 lightcurve (Figure 6, red line, middle panel) and is probably caused by the satellite pointing error of $\pm 1^{\circ}$ which affects the throughput of the light leak. Thus, any given CHIPS solar observation is only good to within a factor of $\approx$ two in absolute flux. Line ratios and correlations, however, are much more reliable since systematic uncertainties and instrumental effects cancel out.

The variations in count rate observed in Figure 6 (upper panel) may be caused by a scale factor that is constant over the passband, or may also have a wavelength dependence. If the scatter is caused by just a scale factor, then it will cancel out when taking line ratios. If, however, there is also a time-varying wavelength dependence in sensitivity, then the degree of correlation in line intensities should decrease as the separation in wavelength between a pair of lines increases.

To determine line intensities, the scale factor needed to match the spectrum in question to the reference spectrum is first determined. This scale factor is then applied to the mean background reference spectrum and each spectrum is then background subtracted. Count rates are next determined in $2 \AA$ wide bins centered on the wavelengths of the desired features. For the correlation analysis, the individual line intensities for each observation are presented as a percentage of total count rate of the entire passband for that observation. Correlation plots are made that compare lines of both the same and different ionization states, and lines both close and far apart in wavelength. Some of the more instructive cases are presented as Figure 7, and fall into three distinct categories. The first group are the strongly correlated lines of Fe IX through Fe XI (dominated by Fe IX 171.073 $\AA$ ) which peak in brightness at a temperature around $1 \mathrm{MK}$ (Figure $7(\mathrm{a}-\mathrm{c})$ ). The second group are the $2 \mathrm{MK}$ ionization states of Fe XIII and XIV (dominated by Fe XIV $211.318 \AA$ ) which also show strong correlation in Figure $7(f-h, j)$. Note that the first two groups are distinctly anti-correlated (Figure 7(e)). When the $1 \mathrm{MK}$ lines are bright, the $2 \mathrm{MK}$ lines are faint, and vice versa. The third set involves Fe XII 193.509 $\AA$ and Fe XII 195.119 ̊, which correlate poorly with either of the first two groups (Figure 7(d, i)). Lastly, there is a set of lines (not shown in Figure 7) that show bimodal distributions (i.e., both positive and negative correlations simultaneously). We interpret these cases as involving blends of both high and low temperature lines (e.g., Fe XIV and Si IX at $227 \AA$ A, Figure 4).

If the variations in intensity between observations are caused by a change in the wavelength dependence of the throughput, then all lines in a given wavelength region would vary in unison regardless of ionization state. This is clearly not the case for these data. The observed variations in count rate of the CHIPS spectra when features from one ionization state are compared to another are caused by real temporal variations in plasma temperature. This effect is best illustrated by combining the count rates of a few of the strongest lines into two temperature groups: The $1 \mathrm{MK}$ "cool” group consists of Fe IX $171.073 \AA$, Fe X

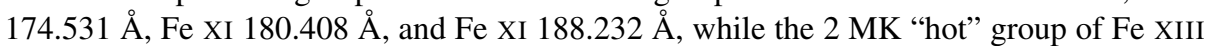

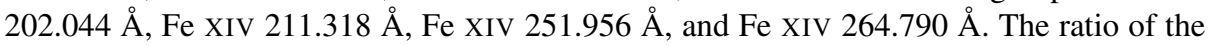
hot-to-cool group count rates is plotted as Figure 6 (dots, middle panel). Any uncertainty in the exposure time or throughput cancels out when taking this ratio. For comparison, the XPSL4 flux is also plotted (red line, middle panel). Evident in the hot-to-cool ratio is the 27day mean rotation period of the Sun (manifested as 27.2-day power in a Fourier spectrum of the hot-to-cool ratio lightcurve), the general decrease in high-temperature activity, and the correlation in slope of the two satellite lightcurves (i.e., when CHIPS sees an increase in temperature, the XPSL4 spectral model shows an increase in flux). However, when the same line ratios are extracted from the XPSL4 spectral model and compared to the CHIPS ratios, the correlation vanishes. This fact indicates that CHIPS is seeing a real change in plasma temperature caused by solar rotation, while the XPSL4 model is just reflecting a 

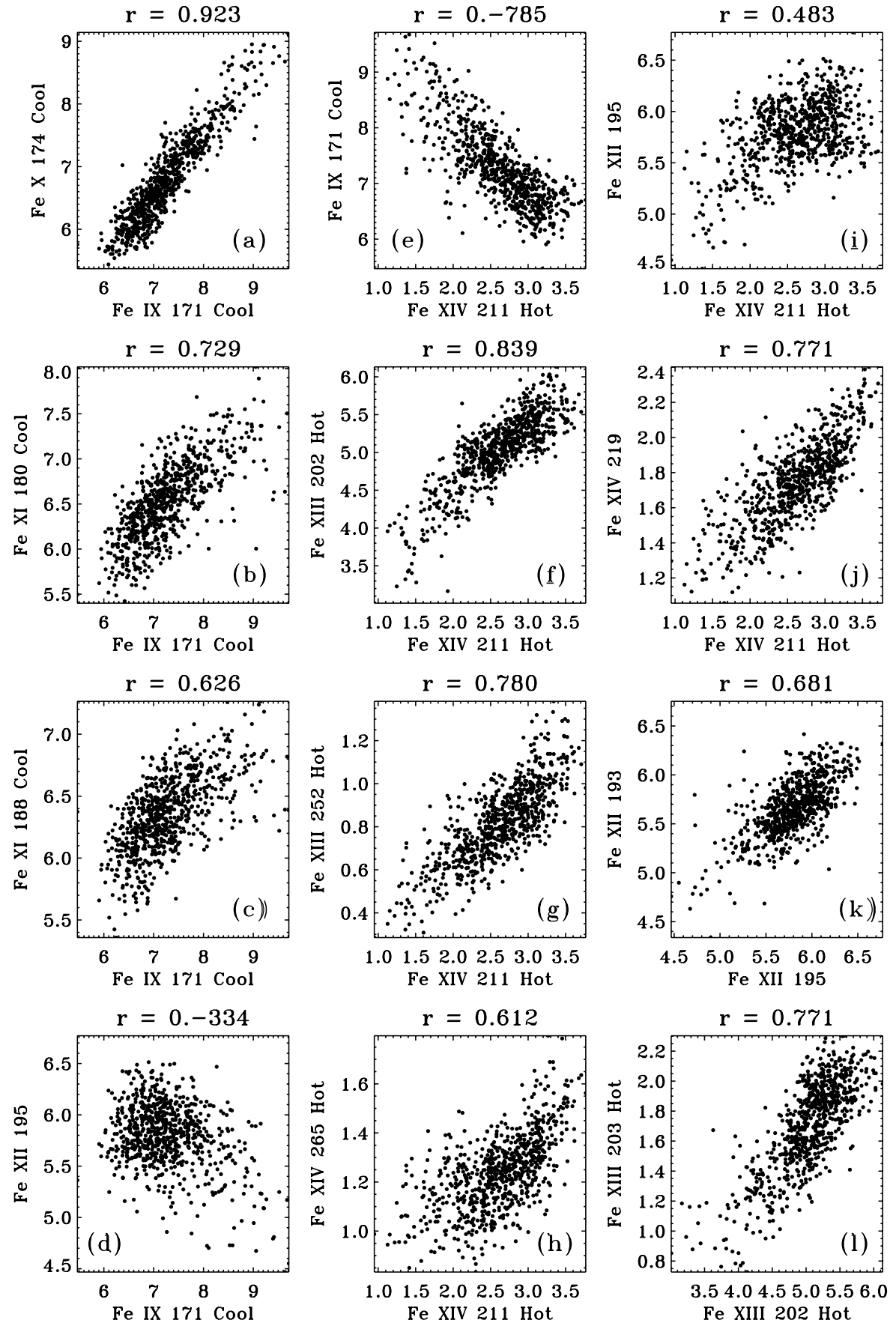

Figure 7 Correlations of Fe-line intensities in units of percent of total Al-filter count rate. Cool vs. Cool $(\mathrm{a}-\mathrm{c})$. Cool vs. Hot (e). Hot vs. Hot (f-h, j). The Fe XII 193 and Fe XII 195 lines are poorly correlated with the Cool (d), and Hot (i) groups, and as well as with each other (k). The density-sensitive Fe XIII 203 and Fe XIII 202 lines are moderately correlated (1). 


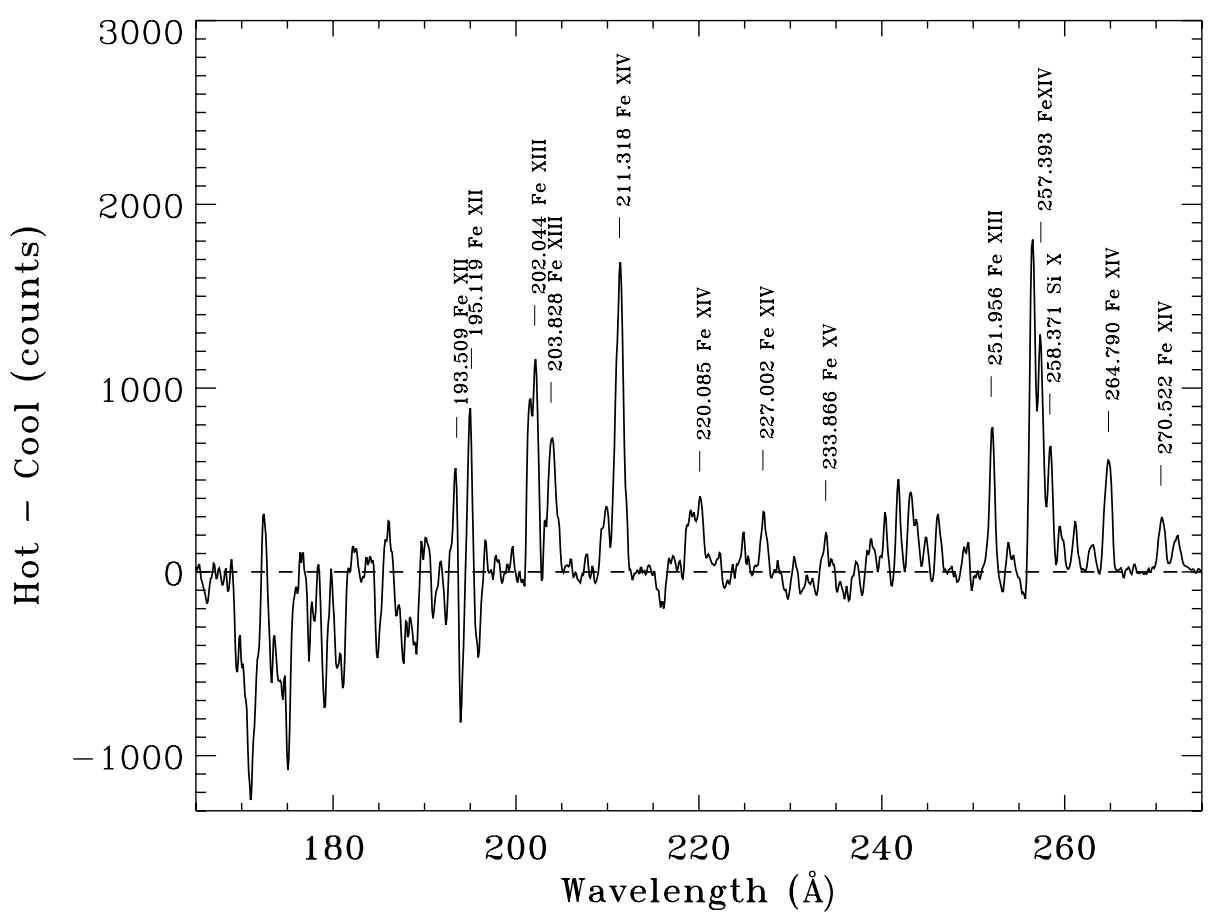

Figure 8 Residuals of the "hot" 2 MK minus "cool" 1 MK spectra. The times from which the hot and cool spectra are created are marked with horizontal dashes in Figure 6 (middle). Only the higher ionization states Fe XII to Fe XV show a significant positive residual. The large negative residuals shortward of $190 \AA$ are from the six bright $1 \mathrm{MK}$ Fe IX - XI lines.

change in overall intensity. In the bottom panel of Figure 6 we plot the density-sensitive line ratio Fe XIII $203.828 \AA / \mathrm{Fe}$ XIII $202.044 \AA$ (which is discussed in Section 5.3), and the International Sunspot Number (red line). Note that the slopes of the CHIPS hot-to-cool ratio, XPSL4 model, sunspot number, and Fe 203/202 ratio are all positively correlated.

To further show that the temporal variations in temperature are real, we construct two spectra filtered on times when the hot-to-cool ratio is at maximum or minimum during 2006 (a period when the XPSL4 lightcurve is relatively flat, Figure 6 (middle)). The "hot" spectrum minus the "cool" spectrum is presented as Figure 8 and shows that only lines with a high ionization state (Fe XII to Fe XV) show a positive residual. Thus, we conclude that the observed hot-to-cool ratio temporal variations are dominated by differences in plasma temperature and not some instrumental effect, and that, because of the observed 27.2-day period, are caused by variations in emission from hotter and cooler regions rotating into, and out of, view. This behavior has also been seen by the TIMED/SEE instrument (Woods et al., 2005).

We also chose two times about 13 days apart $(\approx 1 / 2$, the solar-rotation period) where the hot-to-cool ratio was at minimum or maximum in Figure 6 (diamonds, middle panel), retrieved the corresponding SOHO/EIT full-disk images for the $171 \AA$ and $284 \AA$ channels, and present them as Figure 9. Visual inspection of the images shows no obvious changes in the number and intensity of active regions. However, several independent data sources show an increase in solar activity during this period. The ratio of the SOHO images ( $284 \AA / 171 \AA$ ) 

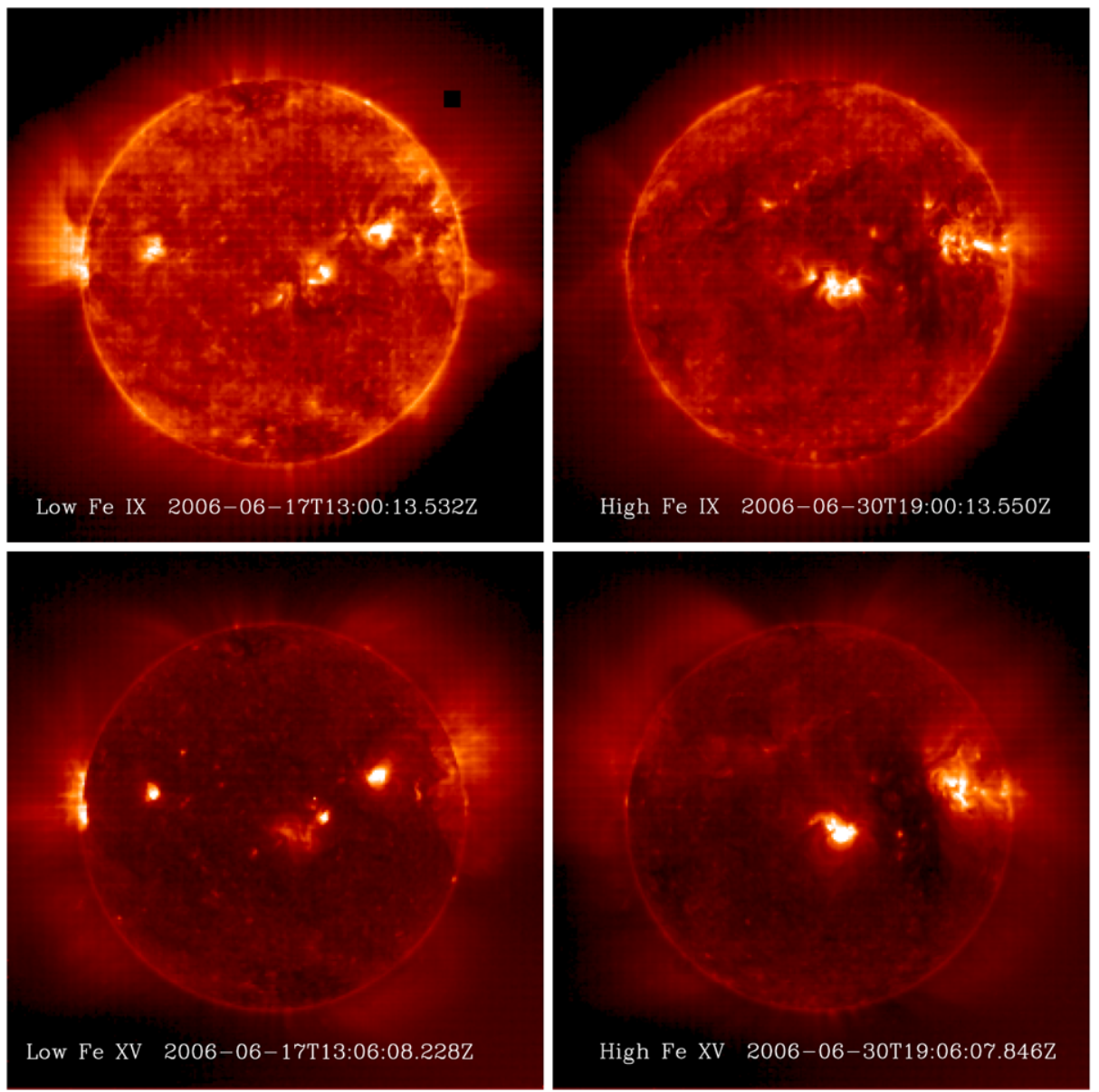

Figure 9 SOHO/EIT images for $171 \AA$ and $284 \AA$ channels for the minimum (left), and maximum (right) hot-to-cool ratio indicated by diamonds in Figure 6.

increased by $25 \%$, the hot-to-cool ratio of CHIPS increased by $50 \%$, and the CHIPS Fe XIII $203 / 202$ ratio by $39 \%$. The sunspot number increased from zero to around 20 during this 13-day period, and the ratio of Michaelson Doppler Interferometer (Scherrer et al., 1995) images extracted for these two times (mdi_fd_2006.06.17_12:51:30.fits, mdi_fd_2006.06.30_17:36:30.fits) shows an increase in magnetic flux by a factor of 1.65 which implies an increase of EUV and X-ray flux (Pevtsov et al., 2003; Fludra and Ireland, 2008). The XPSL4 model shows an increase in total flux of $8 \%$ in the CHIPS passband. These changes are all in the same direction and indicate an increase in solar activity over the 13-day period. The positive correlation between the CHIPS and SOHO/EIT line ratios suggest the possibility of using the CHIPS spectra to help calibrate the SOHO/EIT $171 \AA$ and $195 \AA$ broad-band images, which in turn could be used as proxies to better calibrate other instruments for time periods not covered by CHIPS. We explore these ideas further in Section 6. 


\subsection{Daily Averaged Spectra}

The significant variations seen in Figure 6 are the short-period $(\approx 14$ days) changes in the hot-to-cool line group ratio and the general decrease in intensity of the 2 MK lines over the two-year span of the observations. For reference, we present an atlas of extreme-case hot and cool spectra in Figure 10 that illustrates the transition from moderately active Sun to quiet Sun (compare day 103 to day 825). The hot and cool spectra were extracted during times corresponding to local maxima and minima of the hot-to-cool ratio indicated with + symbols in Figure 6 (middle panel). These spectra are not background subtracted and so illustrate the typical quality of the CHIPS multi-channel contamination-free daily averaged spectra.

\subsection{CHIANTI Modeling}

The CHIPS EUV spectra are from the full solar disk, and as such are the combination of plasma features from the corona and transition region which exhibit large ranges of temperature and density. The dominance of the Fe emission lines, however, allows us to decompose the spectra into several principal components. The overriding strategy is to adequately fit the spectra with the fewest number of atomic elements, temperatures, and densities, and proceeded in this order: identify lines, the peak temperatures associated with the observed lines, and the densities dictated by specific line ratios.

Starting with just Fe, the bulk of the EUV emission is accounted for by a 1 to $2 \mathrm{MK}$ plasma. In this temperature range, a few more features are accounted for by $\mathrm{Si}$ and $\mathrm{Ni}$. The residual flux indicates the presence of $\mathrm{O}$ and $\mathrm{He}$, but at lower temperatures of $\log _{10} T=5.0$ to 5.5. The best density diagnostic available at the CHIPS spectral resolution is the line ratio Fe XIII 203.828 $\AA$ /Fe XIII $202.044 \AA$ whose average value is $0.34 \pm 0.05$ for the entire mission and varied in step with the hot-to-cool ratio (i.e., higher densities are observed when the spectra are hotter, dots on Figure 6, middle and lower panels). This ratio gives an electron density range of $\log _{10} N_{\mathrm{e}}\left(\mathrm{cm}^{-3}\right)=8.5$ to 8.7 which is at the low end of the typical densities determined in and around active regions by the Hinode/EIS (Young et al., 2009; Warren et al., 2008). Fainter lines from other elements (such as $\mathrm{Ne}, \mathrm{Mg}, \mathrm{S}, \mathrm{Ar}$, and Ca seen by the Solar EUV Rocket Telescope and Spectrograph (SERTS): Thomas and Neupert, 1994) were searched for, but could not be identified with any certainty. The spectral resolution of CHIPS is the limiting factor, not the instrument sensitivity.

The standard Differential Emission Measure (DEM) models (quiet Sun, active region, coronal hole) that come with the CHIANTI plasma package version 5.2.1 (Landi et al., 2006; Dere et al., 1997) did not fit the CHIPS spectra very well, either singly, or in combination. We instead built a set of isothermal plasmas combining the elements $\mathrm{Fe}, \mathrm{Ni}$, and $\mathrm{Si}$ (using the solar coronal abundances of Feldman et al. (1992)) at temperatures ranging from $\log _{10} T=$ 5.8 to 6.4 at 0.1 intervals. We chose an average density of $\log _{10} N_{\mathrm{e}}\left(\mathrm{cm}^{-3}\right)=8.6$ since the values determined from observations that ranged from 8.5 to 8.7 made insignificant differences in the model spectra. An isothermal plasma of $\mathrm{He}$ at $\log _{10} T=5.0$, and three of $\mathrm{O}$ at $\log _{10} T=5.3,5.4$, and 5.5 were also constructed, all at a density $\log _{10} N_{\mathrm{e}}\left(\mathrm{cm}^{-3}\right)=10.0$ typical of the transition region (Doschek, 1991). Our model is simply the linear combination of these individual plasmas with the only free parameters being the emission measures.

Because the changes in the CHIPS spectra are large over the short time period of one-half solar rotation, but minor over longer periods, we built two time-averaged spectra corresponding to the minimum and maximum time intervals of the hot-to-cool ratio of Figure 6 (middle panel) during 2006. We fit these hot and cool spectra separately. Good fits were found requiring plasmas of $\log _{10} T=6.0,6.1$, and 6.3. Inclusion of the He plasma decreased $\chi^{2}$ 

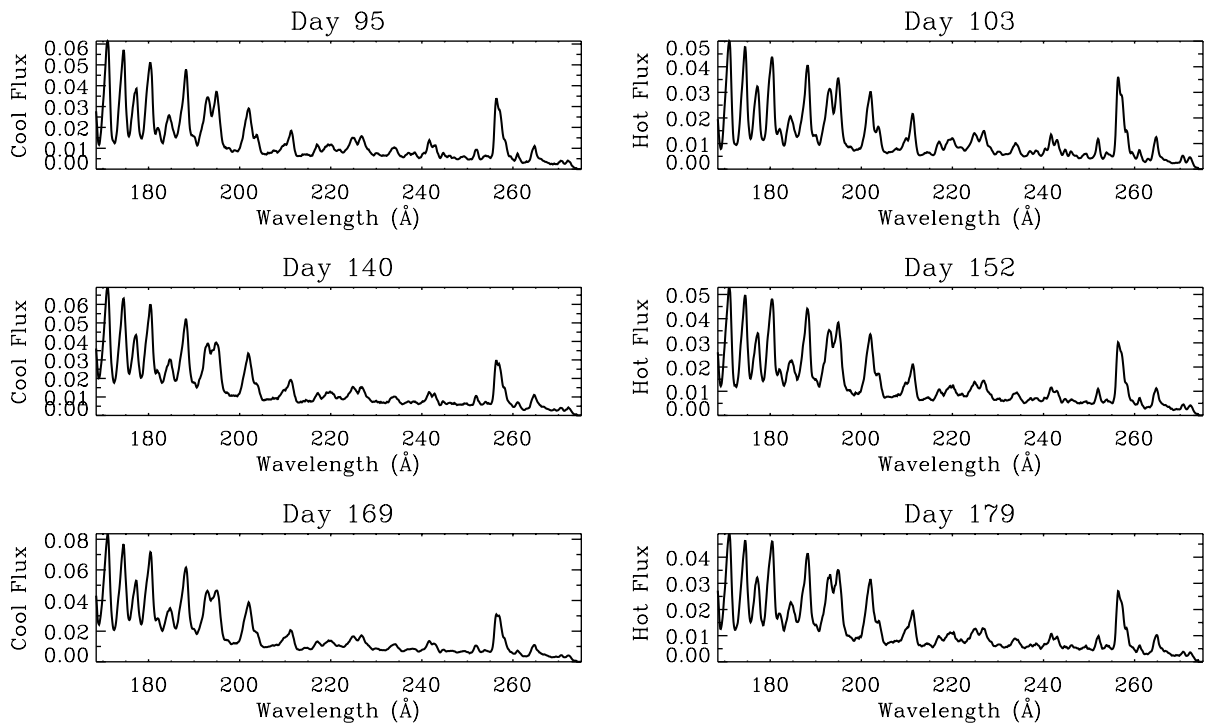

Day 222

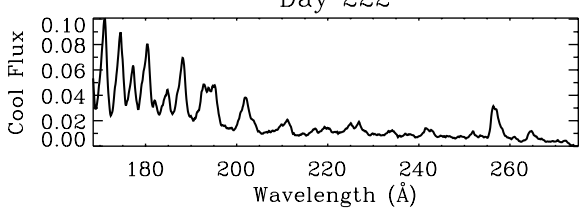

Day 230

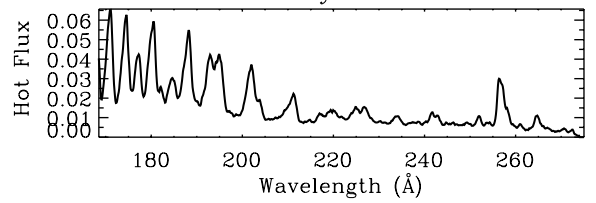

Day 295

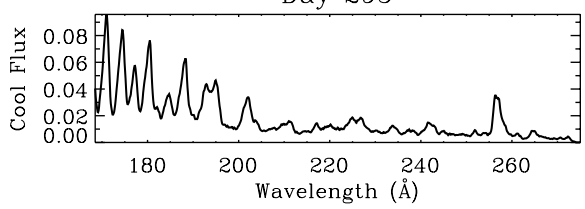

Day 311

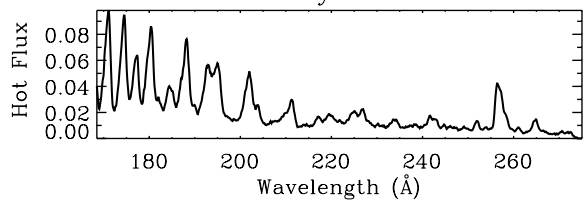

Day 384

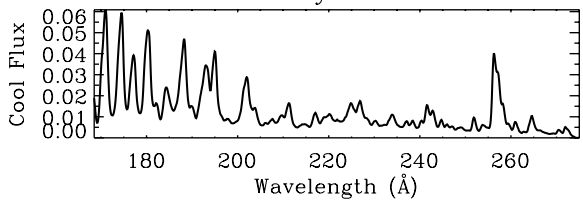

Day 368

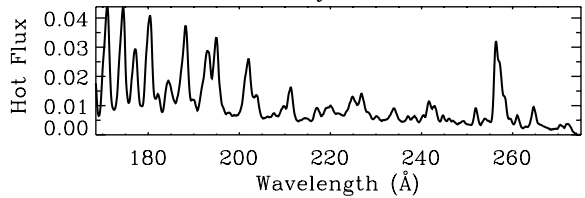

Day 825

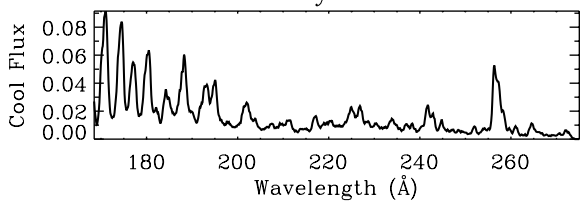

Day 508

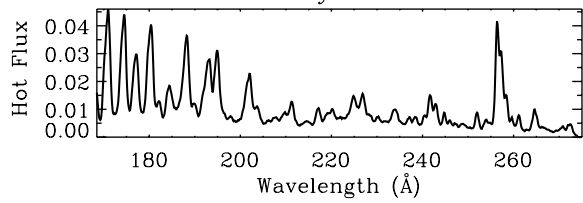

Figure 10 Daily averaged Al-filter spectra covering two years for times corresponding to local extrema of the hot-to-cool ratio (marked with + symbols in Figure 6 (middle)). Cooler spectra are on the left, hotter on the right in units of $\left(\mathrm{erg} \mathrm{s}^{-1} \mathrm{~cm}^{-2} \AA^{-1}\right)$ which are accurate to within a factor of two. 
significantly, while that of $\mathrm{O}$ decreased $\chi^{2}$ by 5 to $8 \%$. The inclusion of the $\log _{10} T=5.8$, $5.9,6.2$, and 6.4 temperature components of the $\mathrm{Fe}-\mathrm{Si}-\mathrm{Ni}$ plasma did not reduce $\chi^{2}$ significantly, and thus are not included in the final CHIANTI model fits.

\subsection{Light-Leak Efficiency}

The modeling outlined in Section 5.3 successfully reproduced the majority of observed features in the CHIPS spectra, but showed a systematic error in amplitude from one end of the spectrum to the other. This residual is an expected consequence of the wavelength dependence of the light leak. The exact nature of the light leak cannot be modeled directly since the angle of incidence and surface roughness of the $\mathrm{Au}$ and $\mathrm{Cu} / \mathrm{Be}$ slit-shutter mechanisms are poorly known. However, the functional form is estimated using the Lawrence Berkeley National Laboratory X-ray Properties of Matter web utility (Henke, Gullikson, and Davis, 1993). We find a smoothly varying function that increases in reflective efficiency with wavelength, and which is well approximated by a simple exponential over the wavelength range of interest

$$
\operatorname{efficiency}(\lambda)=S \exp (\alpha \lambda),
$$

where $\alpha$ governs the wavelength dependence of the light leak, and $S$ is a scale factor, which, when combined with the CHIPS nominal effective-area curves, converts count rate to physical units (determined in Section 5.5).

Our final model is thus the sum of the individual plasmas multiplied by an exponential. The model fits to the cool and hot spectra are shown as Figure 11, and the corresponding emission measures presented in Table 1 . The random errors of the emission-measure determinations are $\approx 8 \%$. The total uncertainty is discussed in Section 6. Since the He and O emissions are from the transition region (Reeves, Timothy, and Huber, 1977) and are optically thick, the CHIANTI-derived values of emission measure may match the data, but are probably only lower limits.

The CHIANTI model fits to the large $\mathrm{Al}$ and $\mathrm{Zr}$ filters produced essentially identical values for temperature, density, and light-leak efficiency. On average, the $\mathrm{Zr}$ data are of inferior quality suffering from multiple-channel contamination and non-uniform slit illumination. Furthermore, because the $\mathrm{Zr}$ passband (140-202 $\mathrm{A}$ ) is smaller than the Al filter, fewer lines are available to constrain the model parameters. However, the presence of $\mathrm{Ni}$ lines in the $\mathrm{Zr}$ filter spectra clearly indicate a plasma component of $\log _{10} T=6.3$, and the relative weakness of the Fe VIII features at $168 \AA$ indicate the absence of significant plasma components of $\log _{10} T=5.8-5.9$. We present only the Al-filter results in Table 1 as they are the most reliable. A new version of CHIANTI (6.0.1) was released in October 2009. The changes, however, do not affect the main conclusions of this work.

\subsection{Comparison to SORCE/XPS and SDO/EVE}

To answer specific questions regarding solar irradiance, solar variability, differentialemission-measure maps, and other solar-physics problems, the CHIPS spectra must be converted to physical units. We accomplish this by determining the average scale factor $[S]$ in Equation (1) by comparing the CHIPS spectra to contemporaneous SORCE/XPS Level 4 model fluxes (Woods et al., 2008) integrated over the passband 145-273 A. We chose the first 370 days since 2006 because during this period the XPSL4 flux remained fairly flat (see Figure 6). The CHIPS ground-based effective-area curves are multiplied by Equation (1) $\left(\operatorname{eff}(145)=6.04 \times 10^{-7}, \operatorname{eff}(275)=3.75 \times 10^{-6}, \operatorname{eff}_{\text {mean }}=1.73 \times 10^{-6}\right)$. We show the 
Figure 11 CHIANTI model fits (dashed lines) to the Al-filter 1 MK "cool" spectrum (solid line, upper panel) and Al-filter $2 \mathrm{MK}$ "hot" spectrum (solid line, lower panel). The reduced $\chi^{2}$ for each fit is also indicated.
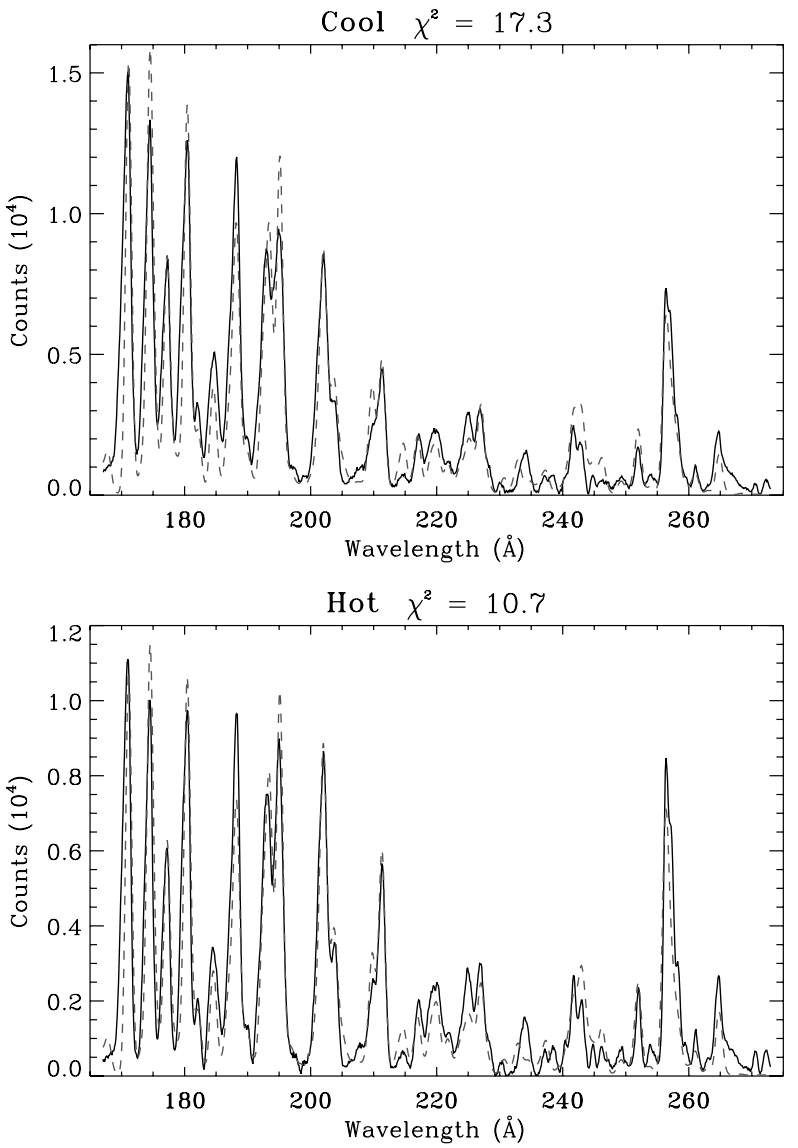

Table 1 Emission measures ${ }^{\mathrm{a}}\left(\mathrm{cm}^{-5}\right)$ determined from CHIANTI modeling of the Al-filter spectra for the different element and temperature plasma components.

\begin{tabular}{lllllll}
\hline Spectrum & $\mathrm{Fe}-\mathrm{Si}-\mathrm{Ni}$ & & & $\mathrm{He}$ & $\mathrm{O}$ & $\chi^{2}$ \\
\cline { 2 - 7 } & 6.0 & 6.1 & 6.3 & 5.0 & 5.5 & \\
\hline Cool & 20.53 & 20.53 & 20.40 & 20.95 & 20.82 & 17.3 \\
Hot & 20.36 & 20.45 & 20.54 & 21.05 & 20.63 & 10.7 \\
\hline
\end{tabular}

${ }^{\mathrm{a}} \log _{10}$ of the emission measure and temperature are given.

Relative uncertainties in the emission measures are typically $8 \%$.

Absolute uncertainties are discussed in Section 6.

Ionization fractions used are from Mazzotta et al. (1998) and solar corona abundances from Feldman et al. (1992).

mean-flux calibrated CHIPS spectrum as Figure 12 (top) and compare it directly to the XPSL4 spectrum extracted for the same time period. The spectral features are in general agreement, but there are several significant differences which we discuss in Section 6.

In Figure 12 (bottom) we show the EVE rocket spectrum of 14 April 2008 (Chamberlin et al., 2009; Woods et al., 2009), and compare it directly to the scaled CHIPS spectra av- 

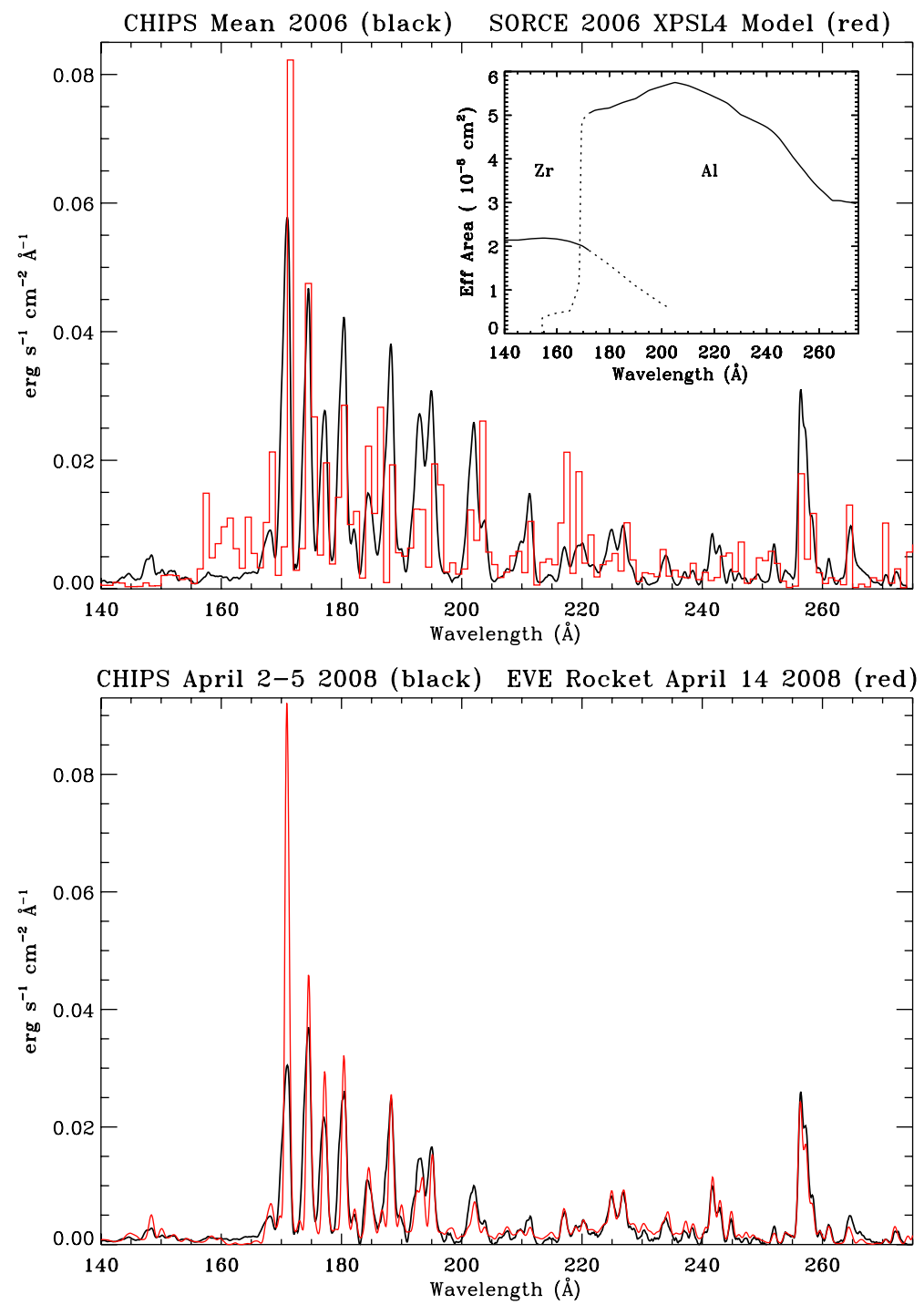

Figure 12 Top: CHIPS spectrum binned at $0.10 \AA$ (black line) scaled to XPSL4 model binned at $1.0 \AA$ (red histogram). The inset plots the nominal effective-area curves of the CHIPS Al and $\mathrm{Zr}$ filters multiplied by the modeled efficiency curve of the light leak. The solid segments of each curve are used for the flux determinations. Bottom: CHIPS spectrum for 2-5 April 2008 (black) scaled to EVE rocket spectrum of 14 April 2008 (red).

eraged over 2-5 April 2008. The excellent agreement between the two spectra shows that both instruments are free from spurious features such as grating ghosts and second-order effects, and that the CHIPS background subtraction and light-leak efficiency determination are reasonable. The only significant difference is the intensity of the Fe IX $171.073 \AA$ line, which we address in Section 6. 
Figure 13 Raw CHIPS

spectrum summed over five days prior to flare binned at $0.11 \AA$ (black) compared to sum of three observations during flare (red).

The only significant difference is some minor multi-channel contamination visible in the 160 to $170 \AA$ region of the flare spectrum.

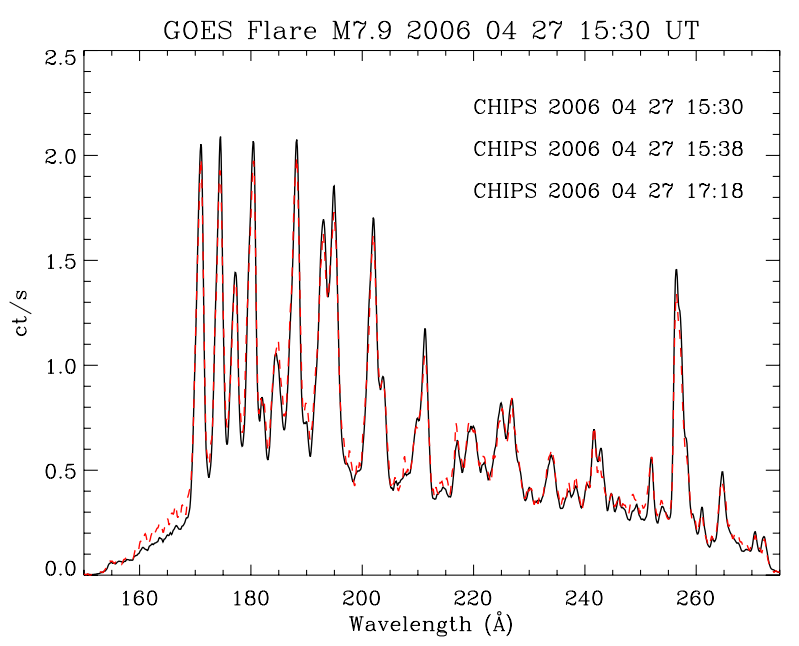

\subsection{Solar Flares}

During 2006, CHIPS observed an M-class flare, and narrowly missed a second flare by 24 hours (GOES X-ray peak times 27 April 2006 15:22 UT M7.9, 06 July 2006 08:13 UT M2.5, respectively). A baseline spectrum was extracted over a five-day period just prior to the first flare and compared to the three CHIPS observations obtained during flare. We present these spectra in Figure 13 in their raw state which is free from any possible systematic error introduced by background subtraction. There is no evidence of a change in plasma temperature. The CHIPS observations of the second flare which were obtained 24 hours after the GOES X-ray peak also showed no differences between the pre- and post-flare spectra.

\section{Discussion and Conclusions}

CHIPS recorded spectra of the solar disk between $140 \AA$ and $273 \AA$ over a two-year period. The general appearance of the spectrum remained relatively stable. The emission lines labeled in Figures 4 and 5 did not appear and disappear, but were visible at all times, even during moderate (M-class) flares and the quiet-Sun period of April 2008. There was, however, variation in the ratio of the hot and cool emission line groups of about $\pm 25 \%$, and a variation in the 1 to $2 \mathrm{MK}$ plasma density of $\pm 15 \%$.

The CHIPS spectra indicate a 1 to 2 MK plasma dominated by Fe emission. Iron is also the most significant element for higher-temperature spectra of active regions and flares, and for wavelengths both shorter and longer than the CHIPS passband (see the rocket-flight spectra of Behring, Cohen, and Feldman, 1972; Malinovsky and Heroux, 1973; Thomas and Neupert, 1994; and the SkyLab data of Dere, 1978). In Table 2 we present the fraction of total intensity for each element used in the CHIANTI fits, and the temperature of peak emission. Recent theoretical work by Colgan et al. (2008) using the Los Alamos ATOMIC plasma code concludes that Fe plays the dominant role in total radiative loss of the corona, even when it is depleted relative to the other elements.

The CHIPS hot and cool spectra are reasonably well modeled with a small set of isothermal plasmas of just five elements, five temperatures, and two densities. The standard DEMs supplied with the CHIANTI package do not model the CHIPS spectra nearly as well. These 
Table 2 Fraction of total intensity by species, and temperature of peak emission between $140 \AA$ and $273 \AA$.

\begin{tabular}{lll}
\hline Element & Fraction $(\%)$ & $\log _{10} T_{\text {peak }}$ \\
\hline $\mathrm{Fe}$ & 85.3 & $6.0-6.3$ \\
$\mathrm{Si}$ & 5.7 & 6.1 \\
$\mathrm{Ni}$ & 1.8 & 6.3 \\
$\mathrm{He}$ & 4.3 & 5.0 \\
$\mathrm{O}$ & 3.0 & 5.5 \\
\hline
\end{tabular}

results are in agreement with those of Cirtain et al. (2006) who, in a study of the unresolved EUV corona, conclude that above non-flaring active regions the corona is isothermal (typically between 1 and $2 \mathrm{MK})$, has an average electron density of $\log _{10} N_{\mathrm{e}}\left(\mathrm{cm}^{-3}\right)=8.0$, and varies little in time. In a subsequent survey of 20 active regions, J. Cirtain (2010, private communication) also finds that the unresolved EUV corona component comprises $55 \%$ of the total EUV emission. We propose that the variation seen by CHIPS in plasma temperature over the solar-rotation period is caused by hotter unresolved plasma associated with active regions, and cooler plasma rotating into and out of view.

The spectral shape, line ratios, temperatures, densities, and light-leak efficiency presented here are determined directly from the CHIPS data. The integrated XPSL4 flux is used only to adjust the CHIPS spectra by a single scale factor (one per observation) when converting count rate to physical flux units. Any CHIPS solar spectrum, when divided by the exposure time and the modified effective-area curves, now yield a calibrated flux ( $\left.\mathrm{erg} \mathrm{s}^{-1} \mathrm{~cm}^{-2} \AA^{-1}\right)$ to within a factor of two if raw, or to within 50\% if scaled to XPSL4 (Woods et al., 2008; Woods, 2010). However, since the XPS CHIANTI model spectrum shows several significant differences when compared to the empirical CHIPS mean solar spectrum, there are probably additional systematic uncertainties in the integrated XPSL4 flux. In particular, the XPSL4 model underpredicts He II, and overpredicts Ni XII and Ni XIII around $160 \AA$ A. Several other spectral features in Figure 12 differ by factors of $\approx$ two. The Ni X and XI lines (seen by CHIPS shortward of $150 \AA$ ) are not yet in the CHIANTI (version 5.2.1) database. In the XPSL4 spectra, Fe IX $171.073 \AA$ is typically twice as bright as its closest neighbors, whereas the CHIPS spectra consistently show it to be only $\approx 8 \%$ brighter. The XPSL4 line ratio Fe XIII $203.828 \AA / F e$ XIII $202.044 \AA$ averaged 2.0 in 2006 which is about a factor of six greater than the value determined from the CHIANTI fits to CHIPS spectra for the same time period. This large discrepancy implies that the electron density used by the XPSL4 model is an order of magnitude larger than that required to model the CHIPS spectra (see Figure 20 in Young et al. (2009)). In general, features visible at temperatures $>\log _{10} T=6.3$, and $<\log _{10} T=6.0$, are overestimated by the XPSL4 model. One important difference to bear in mind between the CHIPS empirical spectra and SORCE XPS Level 4 model is that XPSL4 is based on a single photodiode (Ti filter, 1-70 $\AA$ passband, Woods et al. (2008)), which does not overlap the CHIPS spectral range. So, there is no surprise that there are some differences. These differences may result in systematic uncertainties in the XPSL4 model flux and can be eliminated if the SORCE XPS diode currents are used to scale a CHIANTI model that is based upon DEMs derived from empirical spectra rather than assumed DEMs and out-of-band proxies.

The agreement between CHIPS and EVE seen in Figure 12 (bottom) is excellent apart from the factor of two discrepancy at $171 \AA$. A likely cause for this difference may be that the resolution of the effective-area curve of one of the instruments does not match the actual instrument resolution in the region of the $\mathrm{Al}$ absorption edge around $169 \AA$. To test this idea we compare the Fe IX $171.073 \AA$ line to the Fe X $174.531 \AA$ line since the two lines are close 
together in wavelength and, hence, the difference in instrument throughput is small. Over the course of the two-year CHIPS mission, the 171/174 $\AA$ line ratio averaged $1.09 \pm 0.05$, and $1.07 \pm 0.09$ for the $\mathrm{Al}$ and $\mathrm{Zr}$ filters, respectively. Because the $\mathrm{Zr}$ filter has no absorption edge near $171 \AA$ (see Figure 12, inset), we believe the ratio of near-unity is quite robust. A quietSun spectrum obtained in early 1997 from SOHO/GIS shows a 171/174 $\AA$ line ratio of 1.3 (see Figure 2 of Kuin and Del Zanna, 2007), and the full-disk rocket spectrum of 04 April 1969 (obtained during a period of some solar activity) shows a ratio 1.0 (Malinovsky and Heroux, 1973).

The total uncertainty in absolute flux of the CHIPS spectra is the sum of the XPS absolute photometric calibration uncertainty $(50 \%)$ and the systematic error introduced in scaling the CHIPS spectra to the XPSL4 model. By definition, this latter error is zero when averaged over the entire CHIPS band and over an entire solar-rotation period, but as can be seen in Figure 12 (top) is often a factor of $\approx$ two for individual features. Since the XPSL4 products are at $1.0 \AA$ bin width, a quantitative line-by-line comparison between CHIPS and XPSL4 is currently not possible.

The CHIPS spectra can be used to help cross-calibrate TIMED/SEE, SORCE/XPS, and $\mathrm{SOHO} / \mathrm{EIT}$ for periods of simultaneous observations. Then SOHO/EIT line ratios could be used to determine relative amounts of hot $(2 \mathrm{MK})$ and cool (1 MK) plasma to make a more realistic solar spectrum for the SORCE/XPS CHIANTI models for periods where CHIPS data do not exist.

In summary, our primary findings are as follows.

- Near simultaneous spectra from CHIPS and the SDO/EVE rocket show excellent agreement indicating that both instruments perform as designed, and that the CHIPS light-leak throughput is calibrated correctly.

- Full-solar-disk EUV spectra obtained April 2006 through April 2008 show temporal variations of up to $25 \%$ with a period of 27.2 days. CHIANTI model plasma fits to the spectra show changes in the relative proportions of $1 \mathrm{MK}$ and $2 \mathrm{MK}$ plasmas, and changes in plasma density of $\log _{10} N_{\mathrm{e}}\left(\mathrm{cm}^{-3}\right)=8.5$ to 8.7 over this period.

- The CHIPS spectra are well modeled with a simple set of five isothermal plasmas requiring five temperatures, five elements, and two densities. A 1 to $2 \mathrm{MK}$ plasma of $\mathrm{Fe}$ accounts for $85 \%$ of the observed flux.

- The full-disk spectra are less well fit by standard CHIANTI DEMs which overpredict features above $\log _{10} T=6.3$ and below $\log _{10} T=6.0$. This is true for individual spectra as well as those averaged over many solar rotations and suggests that the true solar DEMs are not smoothly varying functions.

- The CHIPS spectra may be utilized to help cross-calibrate contemporaneous SOHO/EIT, TIMED/SEE, and SORCE/XPS broad-band data, which ultimately will improve our knowledge of the total solar irradiance.

Acknowledgements This work is supported by the Office of Space Sciences, National Aeronautics and Space Administration, under Grant No. NAG5-5219. We thank Brian Welsch for numerous discussions and comments on the manuscript, the anonymous referee for many helpful improvements, Michael Sholl for Figures 1 and 2, Tom Woods and the LASP Team for providing the high-resolution SDO/EVE rocket spectrum, and John McDonald, Jeremy Thorsness, and Mark Lewis of the CHIPS Operations Team who kept the instrument flying for several years beyond its expected lifetime.

Open Access This article is distributed under the terms of the Creative Commons Attribution Noncommercial License which permits any noncommercial use, distribution, and reproduction in any medium, provided the original author(s) and source are credited. 


\section{Appendix}

All CHIPS solar observations will be made available through a public archive a year from the acceptance of this paper. Preliminary mean "hot" and "cool" state spectra may be found at http://ssl.berkeley.edu/chips/archive.html. Each observation is stored in standard FITS table format. The polyamide- $\mathrm{B} / \mathrm{Al}$ and $\mathrm{Zr} / \mathrm{Al}$-filter spectra are presented on a common, uniform $(0.1 \AA)$ wavelength grid as both raw counts, and in flux calibrated units.

The FITS headers contain spacecraft parameters determined at the time of telemetry processing, plus subsequent parameters created during the standard science pipeline processing. Additional parameters unique to the solar observations are also included. Because the EUV emitting region of the Sun extends beyond the solar limb and is quite variable, the Sun is treated as a point source. Hence, flux units of $\left(\mathrm{erg} \mathrm{s}^{-1} \mathrm{~cm}^{-2} \AA^{-1}\right)$ are used, which are good to within a factor of two.

Finally, as a convenience to other researchers, the multiplicative conversion factor required to scale the CHIPS spectra (over the 140-273 A passband) to the contemporaneous SORCE/XPS Level 4 data products is also included for each observation. However, if the XPSL4 products are recalibrated, then this scale factor will also change somewhat.

\section{References}

Behring, W.E., Cohen, L., Feldman, U.: 1972, The solar spectrum: Wavelengths and identifications from 60 to 385 angstroms. Astrophys. J. 175, 493. doi:10.1086/151575.

Chamberlin, P.C., Woods, T.N., Crotser, D.A., Eparvier, F.G., Hock, R.A., Woodraska, D.N.: 2009, New, higher resolution solar extreme ultraviolet (EUV) irradiance results for solar cycle minimum conditions on April 14, 2008. Geophys. Res. Lett. 36, 5102. doi:10.1029/2008GL037145.

Cirtain, J., Martens, P.C.H., Acton, L.W., Weber, M.: 2006, The EUV unresolved corona. Solar Phys. 235, 295 - 316. doi:10.1007/s11207-006-0035-3.

Colgan, J., Abdallah, J.Jr., Sherrill, M.E., Foster, M., Fontes, C.J., Feldman, U.: 2008, Radiative losses of solar coronal plasmas. Astrophys. J. 689, 585-592. doi:10.1086/592561.

Dere, K.P.: 1978, Spectral lines observed in solar flares between 171 and 630 angstroms. Astrophys. J. 221, 1062 - 1067. doi:10.1086/156110.

Dere, K.P., Landi, E., Mason, H.E., Monsignori Fossi, B.C., Young, P.R.: 1997, CHIANTI - an atomic database for emission lines. Astron. Astrophys. Suppl. 125, 149-173. doi:10.1051/aas:1997368.

Doschek, G.A.: 1991, Extreme ultraviolet spectroscopy as a probe of astrophysical plasmas-learning from the Sun. In: Malina, R.F., Bowyer, S. (eds.) Extreme Ultraviolet Astronomy, Pergamon Press, New York.

Feldman, U., Mandelbaum, P., Seely, J.F., Doschek, G.A., Gursky, H.: 1992, The potential for plasma diagnostics from stellar extreme-ultraviolet observations. Astrophys. J. Suppl. 81, 387-408. doi:10. $1086 / 191698$.

Fludra, A., Ireland, J.: 2008, Radiative and magnetic properties of solar active regions. I. Global magnetic field and EUV line intensities. Astron. Astrophys. 483, 609-621. doi:10.1051/0004-6361:20078183.

Fuller-Rowell, T., Solomon, S., Roble, R., Viereck, R.: 2004, Impact of solar EUV, XUV, and X-ray variations on Earth's atmosphere. In: Pap, J.M., Fox, P., Frohlich, C., Hudson, H.S., Kuhn, J., McCormack, J., North, G., Sprigg, W., Wu, S.T. (eds.) Solar Variability and its Effects on Climate, Geophys. Monogr. Ser. 141, AGU, Washington, 341.

Henke, B.L., Gullikson, E.M., Davis, J.C.: 1993, X-ray interactions: Photoabsorption, scattering, transmission, and reflection at $E=50-30000 \mathrm{eV}, Z=1-92$. At. Data Nucl. Data Tables 54, 181. doi:10.1006/ adnd.1993.1013.

Hock, R.A., Eparvier, F.G.: 2008, Cross-calibration of TIMED SEE and SOHO EIT irradiances. Solar Phys. 250, 207 - 219. doi:10.1007/s11207-008-9203-y.

Hurwitz, M., Sasseen, T.P., Sirk, M.M.: 2005, Observations of diffuse extreme-ultraviolet emission with the cosmic hot interstellar plasma spectrometer (CHIPS). Astrophys. J. 623, 911 - 916. doi:10.1086/428446.

Hurwitz, M.V., Sasseen, T.P., Sirk, M., Marchant, W., McDonald, J., Thorsness, J., Lewis, M., Woods, T.: 2006, Extreme ultraviolet solar spectroscopy with CHIPS. Bull. Am. Astron. Soc. 38, 1292.

Kuin, N.P.M., Del Zanna, G.: 2007, The in-flight performance of the SOHO/CDS grazing incidence spectrometer. Solar Phys. 242, 187 -211. doi:10.1007/s11207-007-0306-7. 
Landi, E., Del Zanna, G., Young, P.R., Dere, K.P., Mason, H.E., Landini, M.: 2006, CHIANTI-an atomic database for emission lines. VII. New data for X-rays and other improvements. Astrophys. J. Suppl. 162, 261 -280. doi:10.1086/498148.

Lepson, J.K., Beiersdorfer, P., Hurwitz, M., Sirk, M.M., Kato, T., Yamamoto, N.: 2008, Iron emission lines in solar and laboratory plasmas. Phys. Conf. Ser. 130(1), 012014. doi:10.1088/1742-6596/130/1/012014.

Malinovsky, L., Heroux, M.: 1973, An analysis of the solar extreme-ultraviolet between 50 and $300 \AA$. Astrophys. J. 181, 1009 - 1030 doi:10.1086/152108.

Marckwordt, M.R., Gaines, G.A., Edelstein, J., Hemphill, R., Hull, J.S., Hurwitz, M., Lampton, M.L., McKee, K., Sasseen, T.P., Sholl, M., Siegmund, O.H.W., Sirk, M.M., Taylor, E.R.: 2003, EUV detector of the cosmic hot interstellar plasma spectrometer. In: Siegmund, O.H.W. (ed.) SPIE CS-5164, 43-53. doi:10.1117/12.512279.

Mazzotta, P., Mazzitelli, G., Colafrancesco, S., Vittorio, N.: 1998, Ionization balance for optically thin plasmas: Rate coefficients for all atoms and ions of the elements H to NI. Astron. Astrophys. Suppl. 133, 403 - 409. doi:10.1051/aas:1998330.

Mewe, R., Gronenschild, E.H.B.M., van den Oord, G.H.J.: 1985, Calculated X-radiation from optically thin plasmas. V. Astron. Astrophys. Suppl. 62, 197-254.

Pevtsov, A.A., Fisher, G.H., Acton, L.W., Longcope, D.W., Johns-Krull, C.M., Kankelborg, C.C., Metcalf, T.R.: 2003, The relationship between X-ray radiance and magnetic flux. Astrophys. J. 598, 1387-1391. doi:10.1086/378944.

Reeves, E.M., Timothy, J.G., Huber, M.C.E.: 1977, Extreme UV spectroheliometer on the Apollo Telescope Mount. Appl. Opt. 16, $837-848$.

Sasseen, T.P., Hurwitz, M., Lisse, C.M., Kharchenko, V., Christian, D., Wolk, S.J., Sirk, M.M., Dalgarno, A.: 2006, A search for extreme-ultraviolet emission from comets with the cosmic hot interstellar plasma spectrometer (CHIPS). Astrophys. J. 650, $461-469$. doi:10.1086/507086.

Scherrer, P.H., Bogart, R.S., Bush, R.I., Hoeksema, J.T., Kosovichev, A.G., Schou, J., Rosenberg, W., Springer, L., Tarbell, T.D., Title, A., Wolfson, C.J., Zayer, I., MDI Engineering Team: 1995, The solar oscillations investigation-Michelson Doppler imager. Solar Phys. 162, 129-188. doi:10.1007/ BF00733429.

Sholl, M.: 2005, CHIPS Technical Memorandum 24 October 2005, Space Sciences Laboratory, Berkeley.

Sholl, M., Donakowski, W., Sirk, M.M., Clauss, T., Lampton, M.L., Edelstein, J., Hurwitz, M.: 2003, Optomechanical design of the cosmic hot interstellar plasma spectrometer (CHIPS). In: Blades, J.C., Siegmund, O.H.W. (eds.) SPIE CS-4854, 467 - 478. doi:10.1117/12.460031.

Sirk, M.M., Gaines, G.A., Taylor, E.R., Sholl, M., Marchant, W., Hemphill, R., Kimble, R.A., Sasseen, T., Marckwordt, M.R., Donakowski, W.: 2003, Performance of the cosmic hot interstellar plasma spectrometer. In: Siegmund, O.H.W. (ed.) SPIE CS-5164, 54 - 62. doi:10.1117/12.515601.

Thomas, R.J., Neupert, W.M.: 1994, Extreme ultraviolet spectrum of a solar active region from SERTS. Astrophys. J. Suppl. 91, 461 - 482. doi:10.1086/191944.

Warren, H.P., Ugarte-Urra, I., Doschek, G.A., Brooks, D.H., Williams, D.R.: 2008, Observations of active region loops with the EUV imaging spectrometer on hinode. Astrophys. J. Lett. 686, L131 - L134. doi:10. $1086 / 592960$.

Woods, T.N.: 2008, Recent advances in observations and modeling of the solar ultraviolet and X-ray spectral irradiance. Adv. Space Res. 42, 895 -902. doi:10.1016/j.asr.2007.09.026.

Woods, T.N.: 2010, Private communication.

Woods, T.N., Eparvier, F.G., Bailey, S.M., Chamberlin, P.C., Lean, J., Rottman, G.J., Solomon, S.C., Tobiska, W.K., Woodraska, D.L.: 2005, Solar EUV experiment (SEE): Mission overview and first results. J. Geophys. Res. (Space Phys.) 110, 1312 - 1335. doi:10.1029/2004JA010765.

Woods, T.N., Chamberlin, P.C., Peterson, W.K., Meier, R.R., Richards, P.G., Strickland, D.J., Lu, G., Qian, L., Solomon, S.C., Iijima, B.A., Mannucci, A.J., Tsurutani, B.T.: 2008, XUV photometer system (XPS): Improved solar irradiance algorithm using CHIANTI spectral models. Solar Phys. 250, 235 - 267. doi:10. 1007/s11207-008-9196-6.

Woods, T.N., Chamberlin, P.C., Harder, J.W., Hock, R., Snow, M., Eparvier, F.G., Fontenla, J., McClintock, W.E., Richard, E.C.: 2009, Solar irradiance reference spectra (SIRS) for the 2008 whole heliosphere interval (WHI). Geophys. Res. Lett. 36, 1101. doi:10.1029/2008GL036373.

Young, P.R., Watanabe, T., Hara, H., Mariska, J.T.: 2009, High-precision density measurements in the solar corona. I. Analysis methods and results for Fe XII and Fe XIII. Astron. Astrophys. 495, 587 - 606. doi:10. 1051/0004-6361:200810143. 